\title{
METASTATIC ADENOCARCINOMA OF PARA-URETHRAL GLAND PRESENTING AS INGUINAL LYMPH NODE AND RECURRENT BULBAR URETHRAL STRICTURE
}

\author{
Adam Jones ${ }^{1}$, Samuel Mills ${ }^{1}$, Sinthuja Naguleswaran ${ }^{1}$, Tom Newton ${ }^{2}$, Mohan Pillai ${ }^{1}$ \\ ${ }^{1}$ Department of Urology, East Lancashire Hospitals Trust \\ ${ }^{2}$ Department of Radiology, East Lancashire Hospitals Trust
}

Correspondence: apjones47@doctors.org.uk

Submitted: January 15, 2020. Accepted: February 12, 2020. Published: April 29, 2020.

\begin{abstract}
Para-urethral gland carcinoma is a rare urological malignancy that has a male predominance and has an ageadjusted incidence of 1.5-4.3/million. There are various histological subtypes of para-urethral carcinoma, with adenocarcinoma representing only $16.4 \%$ of these. Treatment is dependent on site, stage and patient factors. A multimodal approach is often adopted for the treatment of this malignancy. This includes radical surgery based on site of malignancy and there have been various case reports describing the role of adjuvant chemotherapy. However, there is still no agreed recommendation or available evidence for treatment of this infrequently encountered malignancy. The majority of patients present with symptoms of advanced disease and outcomes remain poor. We report a case of para-urethral gland adenocarcinoma presenting as recurrent bulbar urethral stricture and inguinal lymph node metastasis. This case report aims to highlight the rarity of the disease and discuss treatment options for this uncommon urological malignancy.
\end{abstract}

Para-urethral gland adenocarcinoma is a rare urological malignancy that is infrequently encountered with an age-adjusted incidence of 1.5-4.3/million. ${ }^{1}$ Patients commonly present with symptoms of advanced disease $^{2}$ and prognosis is poor with overall survival for bulbar cancer at $26 \%{ }^{3}$ Treatment is dependent on the site and stage of the malignancy. Due to disease scarcity, there is no recommended protocol for its management. The authors present a case of paraurethral gland adenocarcinoma presenting as recurrent bulbar urethral stricture and discuss its management.

\section{CASE REPORT}

A 62-year-old male presented to primary care with a painless mass in the left inguinal region and voiding lower urinary tract symptoms (LUTS). His past medical history was for optical urethrotomy for bulbar urethral stricture in 2004, bicuspid aortic valve and hypertension. His drug history was for amlodipine. Examination revealed a palpable 2-cm lymph node $(\mathrm{LN})$ in the left inguinal region. The hernial orifices and external genitalia were normal, digital rectal examination (DRE) revealed a benign prostate. Urine dipstick was negative and routine blood tests were sent. The patient was prescribed antibiotics based on LUTS.

The patient re-presented to primary care with ongoing symptoms and perineal pain. There was no tenderness on repeat DRE. The blood samples taken at the initial presentation were all normal with PSA $2.2 \mathrm{ug} / \mathrm{L}$. The primary care physician arranged for urgent computed tomography (CT) of the abdomen and pelvis.

The CT report commented on enlarged LN measuring $11 \times 21 \mathrm{~mm}$ in the left inguinal region with minor fat stranding. There was no other lymphadenopathy. There was also a thickened bladder wall at 6-7 $\mathrm{mm}$ 
but with no focal bladder lesions. The patient was then referred to the hematuria clinic as a two-week wait.

In the hematuria clinic, the patient described severe voiding LUTS and examination found a palpable blad-der and confirmed the persistent left inguinal LN. He was investigated with flexible cystoscopy that found complete occlusion of the bulbar urethra due to a necrotic mass. The patient had a suprapubic catheter placed for his retention and added to the urology multidisciplinary team meeting (MDT).

At MDT, the CT was re-reviewed and commented on a $35 \times 25 \mathrm{~mm}$ soft tissue intramuscular lesion at the left inferolateral aspect of the pubic symphysis and a further lesion measuring $40 \times 23 \mathrm{~mm}$ at the bulbar urethra. Magnetic resonance imaging (MRI) of the pelvis and CT of the thorax were arranged, in addition to ultrasound-guided biopsy of the LN.

MRI found an abnormal soft tissue lesion with diffusion restriction around the bulbar urethra measuring 45 $\times 28 \mathrm{~mm}$ that infiltrated the apex of the prostate. There was a further $50 \times 36 \mathrm{~mm}$ lesion at the inser-tion of the left obturator externus and adductor brevis muscles and a malignant-looking left inguinal LN (Figure 1). CT thorax was reported as indeterminate opacities in the right lower lobe that required follow up imaging but no definite lung metastases.

Trucut biopsy from the inguinal LN was used to obtain histology. Microscopically, they were part lymphoid tissue and part fibrocollagenous tissue infiltrated by adenocarcinoma (Figure 2). Immunostains were strongly positive for CK-7, CDX-2 and TTF-1 within the metastatic adenocarcinoma. Napscin, CK-20, GATA-3, PSA and thyroglobulin were negative. Following MDT discussion, the patient was referred to oncology for consideration of further treatment.

The patient was reviewed by oncology and had a course of palliative radiotherapy arranged to manage his pelvic and perineal pain. Following radiotherapy, he was seen in the clinic to discuss chemotherapy using the gastro-intestinal agent Oxaliplatin and modified De Gramont (5-Fluorouracil and Folonic acid).

\section{DISCUSSION}

Para-urethral carcinoma (PUC) is a rare type of malignancy. In the United States, the annual age-adjusted incidence is between 1.5-4.3/million. Swartz et al. ${ }^{1}$ reported male predominance to $\mathrm{PUC}$, with men being three times more likely to be affected than females ${ }^{1}$. There are no known causative agents for the development of PUC. Etiological factors include chronic irritation, urethritis, urethral stricture, external beam radiotherapy and human papillomavirus. ${ }^{4}$

PUC can be categorized by histology or location. Swartz et al. reported the histological subtypes of PUC in 1615 patients. This review found transitional cell carcinoma to be most common (55\%), followed by

FIG. 1 Coronal T2 weighted images. A - Mass infiltrating tissue into the fat beneath the right inferior pubic ramus, B - Urethra, C - Mass infiltrating into the base of the left coropora cavernosa.

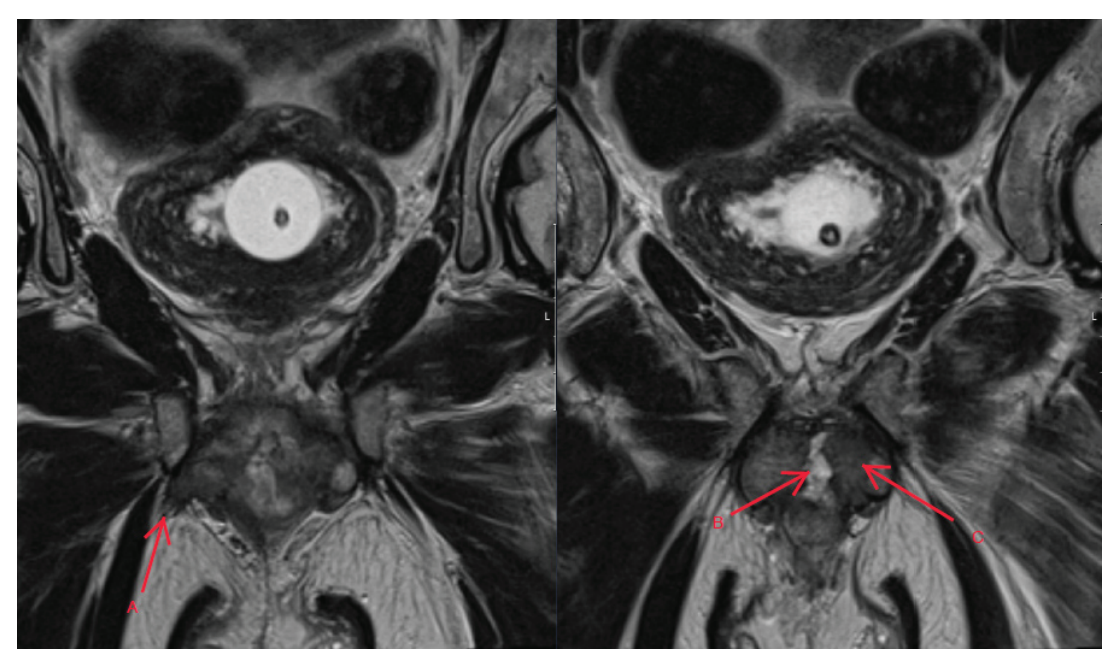

J Endolum Endourol Vol 3(2):1-4; April 29, 2020

This article is distributed under the terms of the Creative Commons Attribution-Non Commercial 4.0 International License. (c) Jones, et al. 
FIG. 2 Core biopsy from the inguinal lymph node is replaced by a moderately differentiated adenocarcinoma composed of atypical glandular structures surrounded by a fibro-collagenous stroma (Hematoxylin and eosin stain $\times 20$ magnification).

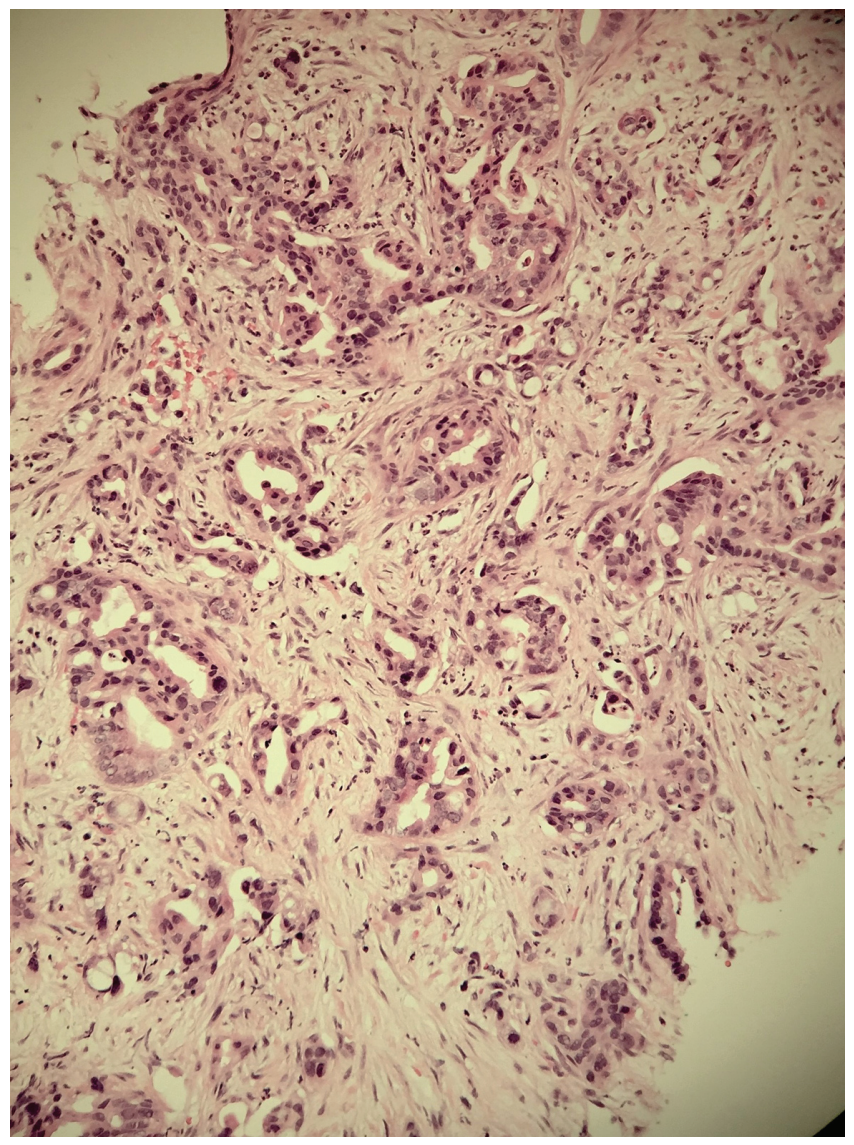

squamous cell carcinoma (21.5\%) and adenocarcinoma $(16.4 \%) .{ }^{1}$ The urethra is an uncommon location for adenocarcinoma. The predominant location for adenocarcinoma of the urinary tract is the bladder, where they often demonstrate mucinous or enteric differentiation. ${ }^{5}$ The most common sites for PUC are the bulbomembranous urethra (60\%) followed by the penile urethra $(30 \%)$ and then the prostatic urethra $(10 \%){ }^{4}$

PUC often presents with symptoms of advanced disease (45-57\%). Other symptoms include hematuria (62\%), extraurethral mass (52\%), obstructive voiding $(48 \%)$, pelvic pain $(33 \%)$ or fistula $(10 \%))^{2}$ These cancers metastasize via a direct extension to surrounding structures or through the lymphatic spread.
Anterior PUC primarily spread to the superficial and deep inguinal lymph nodes. Posterior PUC drain to external, obturator and internal iliac lymph nodes. Palpable inguinal nodes are present in $20 \%$ of PUC. Hematogenous metastasis is rare as is metastasis to distant organs ${ }^{4}$.

Investigations for PUC should include cystoscopy and radiological imaging. Cystoscopy allows for direct visualization of urethral tumour and the opportunity to obtain tissue for histology. Dalbagni et al. ${ }^{3}$ reported that an MRI can provide the best anatomical details, tumour extension and lymph involvement. ${ }^{4}$ MRI may also allow visualization of urethral diverticulum that may contain nodular enhancing malignancy in addition to urethral mass ${ }^{6}$. 
There is no agreed management of PUC adenocarcinoma, ${ }^{4}$ this is as a result of disease rarity. Treatment would be tailored to the disease stage, location and patient factors. A multimodal approach should, therefore, be adopted.

Surgical excision is the preferred option for PUC ${ }^{4}$. For those patients with superficial PUC located within the anterior urethra, surgical options include transurethral resection or distal urethrectomy. In contrast, proximal or advanced PUC may require radical cystoprostatectomy, urethrectomy, and total penectomy. ${ }^{4}$

The effectiveness of chemotherapy is unknown. Gupta et al. reported a case of urethral adenocarcinoma that was treated with radical surgery and adjuvant chemotherapy. The patient received 5-flurouricil and three cycles of cisplatin, which is commonly used for adenocarcinoma for other organs. At the time of publication, Gupta et al. reported no evidence of recurrent disease at 9 months. ${ }^{4}$ In contrast, Gogus et al. reported PUC adenocarcinoma treated with cystectomy and three cycles of methotrexate, vinblastine, epirubicin, and cisplatin but reported recurrence at 5 months and this patient died at 10 months. ${ }^{7}$

\section{CONCLUSION}

We report a rare urological malignancy of the male para-urethral glands presenting as recurrent bulbar urethral stricture and inguinal lymph node metastasis. We consider this case to be unique due to the histological subtype being adenocarcinoma. Treatment for this malignancy is not defined as a result of disease rarity. To the author's best knowledge there is no recommended treatment for this disease and reported treatments are variable. En bloc resection should be considered based on anatomical location and stage. A multimodal approach should be used in advanced cases.

\section{FUNDING AND CONFLICT OF INTEREST}

There was no funding received for the production of this manuscript. No potential conflict of interest was reported by the authorship.

\section{REFERENCES}

1. Swartz MA, Porter MP, Lin DW et al. Incidence of primary urethral carcinoma in the United States. Urology 2006;68:1164-68.

2. Gheiler EL, Tefilli MV, Tiguert R et al. Management of primary urethral cancer. Urology 1998;52(3):487-93.

3. Dalbagni G, Zhang ZF, Lacombe L et al. Male urethral carcinoma: analysis of treatment outcome. Urology 1999;53:1126-32.

4. Gupta R, Gupta S, Basu S et al. Primary adenocarcinoma of the bulbomembranous urethra in a 33-year-old male patient. J Clin Diagnost Res 2017;11:7-8.

5. Stukalin I, Gao Y, Spaner S et al. Primary adenocarcinoma of bulbomembranous urethra: An exceedingly rare carcinoma in a male patient. Urol Case Rep 2019;26:100941.

6. Venyo A. Clear Cell Adenocarcinoma of the Urethra: Review of the Literature. Int J Surg Oncol 2015;790235

7. GöĞUŞ C, Baltaci S, Orhan D et al. Clear cell adenocarcinoma of the male urethra. Int J Urol 2003;10:348-9. 